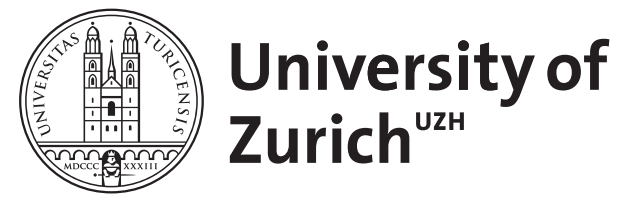

Zurich Open Repository and Archive

University of Zurich

University Library

Strickhofstrasse 39

CH-8057 Zurich

www.zora.uzh.ch

Year: 2001

Genomic imprinting or mutation and interclonal selection in triploid hybrid frogs? A comment on Tunner.

Vorburger, C

DOI: https://doi.org/10.1163/15685380152030472

Posted at the Zurich Open Repository and Archive, University of Zurich ZORA URL: https://doi.org/10.5167/uzh-344

Journal Article

Published Version

Originally published at:

Vorburger, C (2001). Genomic imprinting or mutation and interclonal selection in triploid hybrid frogs?

A comment on Tunner. Amphibia-Reptilia, 22(2):263-265.

DOI: https://doi.org/10.1163/15685380152030472 


\title{
Forum
}

\section{Genomic imprinting or mutation and interclonal selection in triploid hybrid frogs? A comment on Tunner}

\author{
Christoph Vorburger \\ Zoological Institute, University of Zürich, Winterthurerstrasse 190, 8057 Zürich, Switzerland \\ e-mail: chrisvor@zool.unizh.ch
}

In his recent paper, Tunner (2000) investigates genome dosage in triploid hybrid water frogs of the Rana esculenta complex. The hemiclonal frog Rana esculenta is a hybrid between the parental species $R$. ridibunda and $R$. lessonae (Berger, 1967, 1968) and reproduces by hybridogenesi s (Tunner, 1974), i.e. it premeiotically excludes one of its parental genomes from the germline and produces functional gametes containing the unrecombined (clonally transmitted) genome of only one parental species (reviewed by Graf and Polls Pelaz, 1989). Typically, hybrids are diploid and bisexual. Their persistence depends on their co-existence with the parental species whose genome they exclude. In Tunner's study population in the Hungarian plains, $R$. esculenta co-exists with R. ridibunda (R-E-system: Uzzell and Berger, 1975), but the hybrids are unusual in that they are triploid, possessing two L-genomes and one R-genome (LLR-genotype), and all are male. These hybrids exclude the R-genome from their germline and produce diploid spermatozoa containing two L-genomes. Tunner studied several morphological and ecological traits, and examined electrophoretic staining intensities of two proteins with fixed allelic differences between the parental species. He finds that for many (not all) traits, hybrids more closely resemble $R$. ridibunda than $R$. lessonae, although they possess two L-genomes and only one R-genome. Tunner suggests that the hybrids' resemblance to $R$. ridibunda is adaptive in competition for syntopic $R$. ridibunda females, and that genomic imprinting is the best explanation for this unexpected inheritance pattern. I agree with Tunner that a ridibunda-like phenotype may be adaptive in hybrids, but I believe that the presented data do not provide conclusive evidence for the existence of genomic imprinting in triploid $R$. esculenta. Here I explain why, and suggest alternative explanations that may account for the observed pattern.

First, I question whether genomic imprinting can be demonstrated at all in unisexual hybridogens. Tunner uses Reik's (1992) definition of genomic imprinting, "the parent-specific expression or repression of genes or chromosomes in offspring". This definition is broad enough to include the observed pattern, but it may not be useful when applied to unisexual hybridogens, because parentage is confounded with several other factors that may account for differential gene expression. The hybrids in Tunner's study population receive a sexually transmitted, haploid ridibunda chromosome set from their mothers. From their fathers, on the other hand, they receive a diploid lessonae chromosome set which has been transmitted clonally for generations. Obviously, the effect of paternal inheritance on gene expression cannot be separated from possible alternative effects in this system.

Although an overall more lessonae-like phenotype may be the appropriate expectation for hybrids with a LLR-genotype, we would expect mosaic-like variation when looking at a limited set of individual traits. Even in 
the absence of genomic imprinting, some traits would more closely resemble one or the other parental species, and some would be intermediate, depending on their mode of inheritance (McElroy and Kornfield, 1993; Lima et al., 1996). This is exactly what Tunner finds. It remains striking, however, that triploids are similar to $R$. ridibunda in those traits that are likely to affect mating success, like body length, coloration, and breeding time. As Tunner suggests, this bias may be adaptive, because triploid male $R$. esculenta have to compete with $R$. ridibunda males for access to $R$. ridibunda females. Although untested so far, this assumption is certainly plausible: Rana ridibunda females are expected to avoid mating with hybrid males because the resulting offspring would exclude the maternally inherited R-genome from their germline, resulting in zero fitness for the females. A ridibunda-like phenotype is thus likely to increase mating success of triploid $R$. esculenta.

But how does this bias come about? Tunner (p. 139) writes: "The maternal genetic information obviously switches off genetic information encoded in paternal genes in the case of body length, coloration, and certain biological features, such as hibernation, while it does not influence others, i.e. the size of the callus internus." I disagree with this explanation because natural selection cannot act on the "maternal genetic information" in hybrids, which would be necessary for such a mechanism to evolve. Hybrids are a dead end for the maternally inherited R-genomes, because they exclude these genomes from their germline. It is not in the interest of these genomes, nor do they have the possibility to evolve adaptations that increase the mating success of hybrids. Clonally transmitted LL-genomes, on the other hand, have a strong interest in a ridibunda-like phenotype for their carrier, because this increases their chance of transmission and persistence. Such a phenotype may be achieved through repression of gene activity by means of genomic imprinting, but I see three alternative mechanisms that would lead to the same pattern: (i) interclonal selection, (ii) mutation, and (iii) introgression. In areas where triploid hybrids can arise, multiple clonal LL-lineages may be formed and "freeze" some part of the genetic variation available in $R$. lessonae ("frozen niche variation" model: Vrijenhoek, 1979). These lineages will differ in the degree to which they express lessonae-specific traits that are relevant in mate recognition. Where triploid hybrid males compete with $R$. ridibunda males for $R$. ridibunda females, sexual selection by females will favor those clonal lineages with a reduced expression of lessonae-specific traits. The LL-genomes found in mixed populations today may therefore represent a nonrandom subset of those genomes originally "frozen" by hybridization, coding for a more ridibunda-like phenotype. Additionally, nonrecombinant LL-genomes in triploid hybrids can acquire genetic variation through spontaneous mutation (Lynch and Gabriel, 1983). A mutation reducing the expression of a lessonae-specific trait (e.g. causing a loss of the yellow breeding coloration typical for $R$. lessonae) would make the hybrid males' phenotype more ridibunda-like and might be selected for in a mixed population with $R$. ridibunda, because it increases the hybrid males' mating success. Taking these two mechanisms together, I suggest that the clonal LL-genomes of triploid $R$. esculenta in Tunner's study population may be a nonrandom subset of "atypical" lessonae genomes which have accumulated mutations that further reduce the expression of lessonae-specific traits. In principle, this hypothesis is testable. We would expect newly formed triploid hybrid males with a LLR-genotype to have on average a more lessonae-like phenotype than established triploids. Introgression of ridibunda genes into LL-genomes offers an additional explanation for the ridibunda-like phenotype of LLR-triploids. Although hybridogenesi s generally precludes introgression, rare cases of recombination have been documented in R. esculenta (e.g. Uzzell et al., 1977). This possibility is intriguing and warrants further consideration, but it remains purely speculative as long as we do not know whether recombination really occurs in triploid hybrids of the Hungarian plains.

The mechanisms I propose cannot explain the underexpression of the enzyme G3PDH in LL-genomes, which Tunner finds in triploid hybrids. G3PDH is a housekeeping enzyme involved in glycolysis and therefore unlikely to be relevant in mate choice (but for a counterexample see Ward, 2000). But again, it is plausible that mutation accounts for this effect instead of genomic imprinting, especially in hybridogens, where recessive deleterious mutations in clonal genomes are sheltered from selection by the genome of their sexual host (Leslie and Vrijenhoek, 1978). So in summary, mutation and interclonal selection offer a plausible, parsimonious, and in principle testable evolutionary explanation for the overall ridibunda-like phenotype of triploid hybrid water frogs with a LLR-genotype. It cannot be excluded that genomic imprinting accounts for this effect, but Tunner's data do not, in my opinion, provide conclusive evidence for this hypothesis.

Acknowledgements. I wish to thank D.J. Hosken, H.-U. Reyer, B.R. Schmidt, and two anonymous reviewers for helpful comments on the manuscript. 


\section{References}

Berger, L. (1967): Embryonal and larval development of $F_{1}$ generation of green frogs different combinations. Acta Zool. Cracov. 12: 123-160.

Berger, L. (1968): Morphology of the $\mathrm{F}_{1}$ generation of various crosses within Rana esculenta complex. Acta Zool. Cracov. 13: 301-324.

Graf, J.-D., Polls-Pelaz, M. (1989): Evolutionary genetics of the Rana esculenta complex. In: Evolution and Ecology of Unisexual Vertebrates, p. 289-301. Dawley, R.M., Bogart, J.P., Eds, New York, New York State Mus. Bull. 466.

Leslie, J.F., Vrijenhoek, R.C. (1978): Genetic dissection of clonally inherited genomes of Poeciliopsis. I. Linkage analysis and preliminary assessment of deleterious gene loads. Genetics 90: 801-811.

Lima, N.R.W., Kobak, C. J., Vrijenhoek, R.C. (1996): Evolution of sexual mimicry in sperm-dependent all-female forms of Poeciliopsis (Pisces: Poeciliidae). J. Evol. Biol. 9: 185-203.

Lynch, M., Gabriel, W. (1983): Phenotypic evolution and parthenogenesis. Am. Nat. 122: 745-764.

McElroy, D.M., Kornfield, I. (1993): Novel jaw morphology in hybrids between Pseudotropheus zebra and Labeotropheu s fuelleborni (Teleostei, Cichlidae) from Lake Malawi, Africa. Copeia 1993: 933-945.

Reik, W. (1992): Imprinting in leukaemia. Nature 359: 362-363.

Tunner, H.G. (1974): Die klonale Struktur einer Wasserfroschpopulation. Z. zool. Syst. Evolut.-forsch. 12: 309314.

Tunner, H.G. (2000): Evidence for genomic imprinting in unisexual triploid hybrid frogs. Amphibia-Reptilia 21: 135-141.

Uzzell, T., Berger, L. (1975): Electrophoretic phenotypes of Rana ridibunda, Rana lessonae, and their hybridogenetic associate, Rana esculenta. Proc. Acad. Nat. Sci. Philadelphia 127: 13-24.

Uzzell, T., Günther, R., Berger, L. (1977): Rana ridibunda and Rana esculenta: A leaky hybridogenetic system (Amphibia, Salientia). Proc. Acad. Nat. Sci. Philadelphia 128: 147-171.

Vrijenhoek, R.C. (1979): Factors affecting clonal diversity and coexistence. Am. Zool. 19: 787-797.

Ward, P.I. (2000): Cryptic female choice in the yellow dung fly Scathophag a stercoraria (L.). Evolution 54: $1680-1686$

Received: August 8, 2000. Accepted: September 26, 2000. 